\title{
Towards an understanding of the molecular mechanisms of endometriosis-associated symptoms (Review)
}

\author{
SHOGO IMANAKA, SACHIYO MARUYAMA, MAI KIMURA, MIKA NAGAYASU and HIROSHI KOBAYASHI \\ Department of Obstetrics and Gynecology, Nara Medical University, Kashihara, Nara 634-8522, Japan
}

Received April 16, 2020; Accepted June 1, 2020

DOI: $10.3892 /$ wasj.2020.53

\begin{abstract}
Currently, several endometriosis classification systems are available in clinical practice; however, no single classification system can predict the severity of all pre- and post-operative symptoms of endometriosis, such as pain, infertility and fibrosis. The aim of the present review was to reconcile the concepts of endometriosis classification and the molecular aspects of endometriosis. Each keyword alone or in combination was used to search by applying the filters of the title and the publication years between January, 2000 and December, 2019. Several classifications systems have been proposed to categorize the severity of endometriosis. The cause of pain is not only related to anatomic abnormalities, but also to the imbalance between sensory and sympathetic innervation due to the abnormal secretion of a variety of cytokines. Endometriosis-related infertility is a group of diseases manifesting with various morphological and functional abnormalities that negatively affect fertility. Diagnostic imaging, which primarily provides morphological information, has limitations as disease progression is not associated with the prevalence and severity of infertility. Changes in the microenvironment caused by endometriosis-induced inflammation and oxidative stress can lead to impaired fertility. In particular, proinflammatory mediators (cytokines, interleukins and immune dysfunction), oxidative stress markers (hemoglobin, heme, free iron, ROS and antioxidants), hormonal imbalance, proteolytic enzymes and soluble adhesion molecules all may be potential markers for predicting infertility. The major cell types involved in the development of endometriosis-associated fibrosis are platelets, various inflammatory cells, such as macrophages, T lymphocytes, B lymphocytes and natural killer (NK) cells, ectopic endometrial cells, and sensory nerve fibers. A variety of symptoms, such as pain, infertility and fibrosis, involve interrelated signaling mechanisms. Beyond the structural and functional changes that characterize a
\end{abstract}

Correspondence to: Professor Hiroshi Kobayashi, Department of Obstetrics and Gynecology, Nara Medical University, 840 Shijo-cho, Kashihara, Nara 634-8522, Japan

E-mail: hirokoba@naramed-u.ac.jp

Key words: endometriosis, classification, severity, symptoms variety of symptoms, there may be interrelated, common signaling pathways. The present review highlights the molecular mechanisms involved in endometriosis-associated symptoms such as pain, infertility and fibrosis. In order to create a disease severity scoring system that can predict each symptom of endometriosis, not only anatomical findings, but also biochemical parameters, such as oxidative stress are required.

\author{
Contents \\ 1. Introduction \\ 2. Literature search \\ 3. Characteristics of the relevant studies \\ 4. Role of diagnostic imaging in evaluating the severity \\ of endometriosis \\ 5. Pros and cons of existing endometriosis classification \\ systems \\ 6. An update on the endometriosis-associated pain symptoms \\ 7. Endometriosis-associated infertility: An update on \\ clinical aspects \\ 8. Endometriosis-associated infertility: An update on \\ molecular aspects \\ 9. Endometriosis-associated fibrosis: An update on \\ molecular aspects \\ 10. Conclusion and future perspectives
}

\section{Introduction}

Endometriosis is one of the most common gynecological diseases, with a rate of $\sim 10 \%$ of women of reproductive age and $\sim 50 \%$ of those with infertility $(1,2)$. It is characterized by the presence of endometrial tissue outside the uterine cavity (2). The main symptoms are commonly associated with chronic pelvic pain and infertility. Women affected suffer from dysmenorrhea (79\%), pelvic pain (69\%), dyspareunia (45\%), modified gut transit (constipation, diarrhea in 36\%), intestinal pain (29\%), infertility (26\%), ovarian mass $(20 \%)$, dysuria (10\%) and other urinary disorders $(6 \%)(1,3,4)$.

The diagnosis of endometriosis is based on clinical suspicion, a pelvic examination, ultrasound and magnetic resonance imaging (MRI); however, laparoscopy, an invasive surgical procedure, currently remains gold standard for diagnosis (5). 
The preferred diagnostic methods other than laparoscopic inspection are transvaginal ultrasonography (TVS) as the first line option. Endometriotic lesions were classified into 3 phenotypes: Superficial peritoneal endometriosis, ovarian endometrioma and deep infiltrating endometriosis (DIE). Superficial endometriotic foci are difficult to diagnose not only by TVS, but also by MRI and are usually identifiable only at laparoscopy. Therefore, TVS is the first-line imaging technique for the diagnosis of ovarian endometrioma and DIE (6). Several studies have demonstrated the diagnostic accuracy and reliability of TVS for the detection of DIE and pouch of Douglas obliteration (7). In clinical practice, physicians not only diagnose the presence of endometriosis, but also evaluate the spread of the disease and the severity of the symptoms. Usually, the severity of the disease burden is determined based on the location, diameter and depth of the lesions, the presence of deep utero-sacral nodules and the density of adhesions, such as complete obliteration of the cul-de sac. Endometriosis-related pain symptoms may be associated with the severity of diseases, such as adhesions and DIE. In addition, adhesion and fibrosis with anatomic abnormalities will certainly lead to infertility, but even minimal and mild endometriosis is often associated with infertility (8). Sanchez et al found that in vitro fertilization (IVF) outcomes in women with endometriosis indicated that minimal/mild disease has a lower fertilization rate than moderate/severe disease (9). It is often found that the severity of the symptoms is not always associated with the spread or extent of endometriosis. Furthermore, the lack of an association between endometriosis-related pain and infertility makes it difficult to determine the disease severity by imaging alone.

Although the etiology of endometriosis remains unknown, the proposed hypothesis is based on 3 main theories: Retrograde menstruation, coelomic metaplasia and Müllerian remnants (2). Given the different contexts in which endometriosis develops, a single etiological model is not enough to explain its pathogenesis (10). Recent advances and controversies in the etiology, pathogenesis, diagnosis, classification and treatment of endometriosis have been previously discussed in detail (11). Genetics, epigenetics and the related signaling pathways have been implicated in the pathogenesis of endometriosis, and there has recently been interest in inflammation, oxidative stress, iron metabolism, macrophages, platelets, sensory nerve fiber and fibrosis $(2,10)$. It is now accepted that oxidative stress plays an important role in the initiation and progression of endometriosis (12). Several basic and clinical studies have been conducted on the effects of oxidative stress on the endometriosis-related pain, male and female infertility, and the development of fibrosis $(2,10,13-15)$.

Currently, several endometriosis classification systems are used in clinical practice. The classification systems are mainly used to determine the extent of the anatomic abnormality; however, the drawback is that they are not always associated with the severity of the clinical symptoms. The present review compares the strengths and weaknesses of the existing classification systems and discusses their association with the severity of the symptoms. The molecular mechanisms that trigger endometriosis-related symptoms, such as pain, infertility and fibrosis are also reviewed. The final aim of the present review
Table I. Number of articles hit by searching for each keyword alone or in combination.

\begin{tabular}{lrc}
\hline Key words & No. of refs. & No. of eligible articles \\
\hline Endometriosis & & \\
\hline Classification & 778 & \\
Pain & 281 & 29 \\
Infertility & 202 & 19 \\
Fibrosis & 41 & 11 \\
Severity & 2,175 & \\
Pain & 1,021 & \\
$\quad$ Molecular & 57 & 22 \\
Infertility & 651 & 19 \\
$\quad$ Molecular & 58 & \\
Fibrosis & 113 & 6 \\
$\quad$ Molecular & 8 & 18 \\
Inflammation & 124 & 4 \\
Oxidative stress & 28 & \\
Iron & 4 & \\
\hline
\end{tabular}

Each keyword alone or in combination was used to search by applying the filters of the title and the publication years between 2000 and 2019. The number of articles searched by keywords and the number of eligible articles among these are shown. For example, the number of articles hit by searching with the keywords 'endometriosis' AND 'classification' AND 'pain' is 281. Among the 281 articles, 29 articles were eligible.

was to identify the biological mediators that contribute most to the symptoms of endometriosis.

\section{Literature search}

A computerized literature search was conducted to identify relevant studies reported in the English language. A comprehensive literature search was conducted on the PubMed and Embase databases between January, 2000 and December, 2019, combining the keywords 'endometriosis', 'classification', 'severity', 'pain', 'infertility', 'fibrosis', 'molecular', 'inflammation', 'oxidative stress' and 'iron'. A variety of combinations of these terms were used, depending on which database was searched (Table I). Furthermore, the references of each article were searched to identify potentially relevant studies. Publications of original studies, review articles and some guidelines were included, while those documenting opinions, points of view or anecdotes were excluded.

\section{Characteristics of the relevant studies}

A selection flow chart for the search strategy is presented in Fig. 1. Based on the search strategy, a total of 778 and 2,175 articles were initially identified on 'endometriosis' and 'classification' (Fig. 1, left panel) and 'endometriosis' and 'severity' (Fig. 1, right panel), respectively. Following the initial review of titles and abstracts, 121 and 815 articles were retained, respectively. 


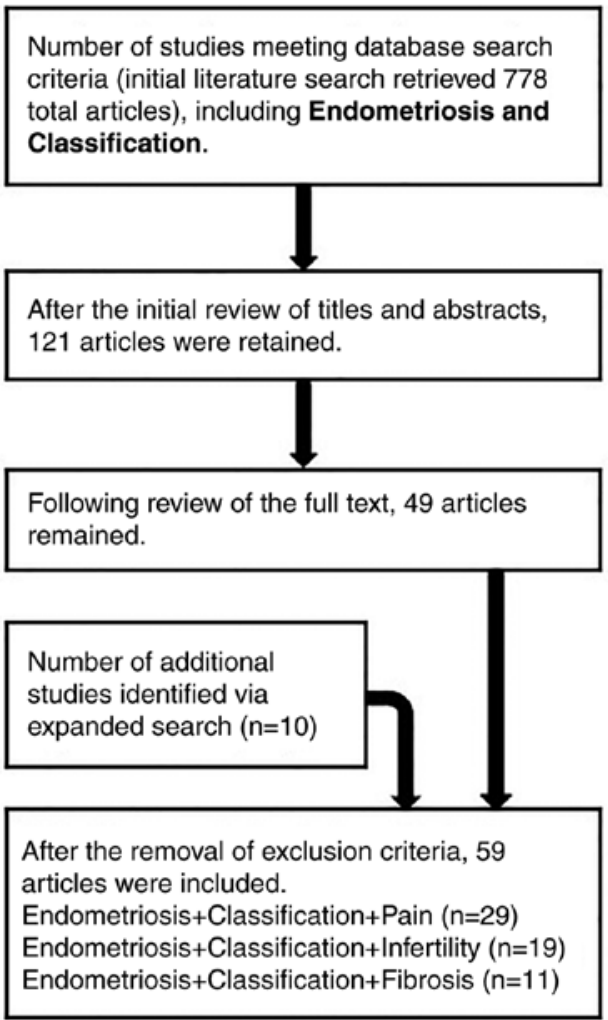



Figure 1. Flow chart of the literature search of the present review article.

Following the review of the full text, 49 and 111 articles remained, respectively, also following the removal of opinions, points of view or anecdotes. The number of additional studies identified by an expanded search was 10 and 13, respectively. Finally, 59 and 124 articles were included, respectively.

\section{Role of diagnostic imaging in evaluating the severity of endometriosis}

Laparoscopy remains the gold standard for the diagnosis of endometriosis, although it is preferably diagnosed in a non-invasive manner (16). It is quite difficult to identify superficial diseases, peritoneal lesions, or early/mild deep endometriosis by imaging modalities $(17,18)$, indicating that a negative diagnosis cannot rule out endometriosis. On the other hand, TVS and MRI are useful in detecting advanced stages of endometriosis. Endometriosis with adhesions to surrounding organs, e.g., extensive inflammatory adhesions between ovarian endometrioma and the rectum, is considered severe. The items for determining adhesions in TVS using the dynamic manipulation of the pelvic organs are the loss of ovarian mobility and limited sliding between the posterior uterine serosa and the bowels (17). Holland et al revealed that the overall sensitivity and specificity of TVS for the diagnosis of severe endometriosis were 85 and 98\%, respectively (17). Women with TVS features of ovarian endometriomas have greater ovarian immobility than do women without these features, with the sensitivity and specificity of 89 and $90 \%$, respectively $(19,20)$. As the ability of MRI to diagnose adhesions or complete obliteration of the pouch of Douglas is similar to that of dynamic TVS, there is no need to perform routine MRI following TVS (7). Therefore, the diagnostic accuracy of dynamic TVS is comparable with, and may be superior to, routine MRI $(17,18)$. However, MRI has the better advantage of objectivity and reproducibility than TVS. Based on the above, TVS and MRI are valuable tools for the diagnosis of the severity of endometriosis, including adhesions; it may be possible to develop a classification for endometriosis-related pain. On the other hand, there is a major unsolved issue regarding endometriosis-related infertility. Imaging diagnostics, which primarily determine the presence of morphological abnormalities, have limitations as disease progression is not associated with the prevalence and severity of infertility (21). Several research groups have developed a simplified classification to diagnose the severity of endometriosis-related infertility (discussed below). The adhesion scoring system created by Ichikawa et al can predict post-operative adhesion and infertility (22). The scoring system also allows for the selection of patients to undergo IVF following surgery (22). Research on the association between the progression of endometriosis (spreading and adhesion) and the severity of symptoms (pain and infertility) is a topic for the future.

\section{Pros and cons of existing endometriosis classification systems}

The various classification schemes for endometriosis staging developed by several professional organizations have been proposed based on anatomic location and disease 
severity $(3,23-26)$. The classification system should at least be applied to various endometriosis structures, lesion appearance, pelvic adhesions and anatomic locations to determine the disease severity. First, the advantages of the revised American Society for Reproductive Medicine (r-ASRM) classification are its worldwide use, familiarity and its easy integration into a number of other classification systems (27). Second, the ENZIAN classification is excellent for the assessment of DIE $(3,24)$. Third, the Endometriosis Fertility Index (EFI) classification can be applied to the prediction of fertility (28). This chapter mainly outlines the advantages and disadvantages of the r-ASRM classification, the ENZIAN classification, the EFI classification and the American Association of Gynecological Laparoscopists (AAGL) classification.

The r-ASRM classification. In 1979, the American Fertility Society (AFS) proposed a novel classification system that links the surgical findings of endometriosis with fertility (29). This classification system was revised in 1985 and became the current edition when the name of the society was changed to the American Society for Reproductive Medicine (r-ASRM) in 1996 (29). The r-ASRM score includes the following items: Peritoneal lesions, ovarian lesions, posterior cul-de-sac obliteration and adnexal adhesions (30). The total score is calculated by adding points according to the size of endometriotic lesions and the extent of adhesions, and the total points are classified into stages I to IV (I, minimal; II, mild; III, moderate; and IV, severe) $(23,29,31)$. Women with minimal or mild endometriosis have superficial implants with very few adhesions, whereas moderate or severe endometriosis is generally characterized by ovarian endometriomas with more severe adhesions. The main color categories of peritoneal endometriosis are classified into red (R), black (B) and white (W) (29). The r-ASRM classification system is standardized, widely used and accepted worldwide as a global standard for almost all clinical studies $(3,23,29)$.

This classification system has some limitations, however (29). There is no association between clinical symptoms and surgical findings due to the lack of assessment of DIE and pain $(29,31,32)$. Even women presenting with minimal clinical symptoms may have advanced disease. It has been pointed out that the advanced stage is not related to the prognosis of infertility, as this classification is less relevant for post-operative natural pregnancy rates $(29,31,32)$. Thus, even women with very few endometrial lesions may have infertility. However, as the advanced stages of endometriosis are associated with a worse prognosis for IVF treatment compared to the early stages or tubal factor infertility, the r-ASRM classification is useful in predicting the IVF outcome (33). More specifically, the disadvantages of this classification are the following: i) Staging is not fully associated with morphological changes in organs; ii) a lack of assessment of retroperitoneal changes and DIE; iii) poor prediction of pregnancy success following treatment; and iv) limited reproducibility (23). Moreover, endometriosis-related pain and infertility are poorly associated with the duration of the disease (23). Therefore, the clinical use of this classification poses significant challenges, particularly in predicting fertility and selecting therapeutic modalities (32). Several studies have searched for candidate biomarkers (blood, ascites, cyst fluid, follicular fluid and tissue that reflect the severity of the symptoms (please see chapter 8 below entitled 'Endometriosis-associated infertility: An update on molecular aspects.'). In the future, a new classification system consisting of anatomic abnormalities and these biomarkers will need to be developed.

The ENZIAN classification. A working group meeting was held in Enzian, Austria in 2005, mainly to create a new classification (the ENZIAN classification) that includes retroperitoneal diseases and DIE $(3,24)$. In 2011, the ENZIAN classification system was reviewed (3). Since DIE can invade into surrounding structures, similar to cancer, this classification was prototyped based on the TNM classification of cervical cancer (25). The ENZIAN score was classified into A (rectovaginal space and vagina), B (sacrouterine ligaments, cardinal ligaments, pelvic sidewall and external ureter compression) and $\mathrm{C}$ (rectum) compartments. The depth of endometriosis invasion was classified as level $1(<1 \mathrm{~cm}$ depth), level $2(1-3 \mathrm{~cm})$ and level $3(>3 \mathrm{~cm})(25)$. The mode of invasion into other organs was also subclassified as FA for adenomyosis, FB for bladder involvement, FU for intrinsic ureter involvement, FI for intestinal involvement and FO for involvement of other organs or structures, such as the abdominal wall $(3,24)$. For patients with DIE, the ENZIAN classification system should be used to completely describe the surgical findings, along with the r-ASRM classification (34). The ENZIAN scores can be viewed at www.endometriose-sef.de/dateien/ENZIAN_2013_web. pdf. The historical background of the ENZIAN classification has been previously described $(23,35)$.

This classification has advantages and disadvantages. Pain and dysmenorrhea are strongly associated with the severity grade in this classification (35). The most affected compartment is the posterior area $(93.4 \%)$, mainly on the left side $(67.8 \%)$, which supports the clinical data that DIE is more likely to invade the sigmoid colon (25). For the evaluation of DIE, attempts have been made to incorporate the ENZIAN classification into the r-ASRM classification $(26,35)$. Due to the poor association between the ENZIAN score and fertility, this score cannot be used to predict the prognosis of endometriosis-related infertility (34). However, it is currently used in German-speaking countries, although it is not widely used internationally (24).

The EFI. In 2010, the EFI was published $(3,28)$. This index has been validated to predict the pregnancy rate of infertile patients after surgical diagnosis and treatment of endometriosis $(3,28)$. This index is useful for planning post-operative assisted reproductive technology (ART) due to its excellent evaluation of pregnancy prognosis (28). The EFI score includes historical factors (age of the patient, duration of infertility and a history of pregnancy) and surgical factors [least function (LF) score at conclusion of surgery, AFS endometriosis score and AFS total score] (28). This score is rated from 0 to 10 , with 0 being the worst prognosis and 10 being the optimal (28). When patients with endometriosis are classified into 3 groups using the EFI score, there is a significant difference in the cumulative pregnancy rate between the groups. The EFI score allows for the prediction of non-ART pregnancy in surgically-treated patients with endometriosis $(3,28)$. On the other hand, there is no significant difference in the cumulative pregnancy rate when 
divided into 4 groups by the r-ASMR classification $(29,31,32)$, indicating that the severity of the r-ASRM classification is not associated with the pregnancy rate.

The AAGL classification. Currently, members of the American Association of Gynecological Laparoscopists (AAGL) Society are developing a classification system that focuses on endometriosis-related infertility and pain $(3,34)$. Based on the results of preliminary studies demonstrating a better association between the AAGL classification system and infertility, pain levels and surgical difficulties, this classification may be used in place of the r-ASRM classification system in the future (34). The document can be obtained from the URL (http://www.aagl. org/wp-content/uploads/2013/03/NewsScope_Oct-Dec_2012. pdf).

Currently, the 4 classifications can be used in various clinical settings. In summary, no single classification can predict the severity of all pre- and post-operative symptoms of endometriosis, particularly pain and fertility. The most important factors are as follows: The classification needed should preferably be simple, easy to use, reproducible, and taking into consideration symptoms, such as pain and infertility, and predicting prognosis.

\section{An update on the endometriosis-associated pain symptoms}

Endometriosis is often found in women with unexplained pelvic pain as it is associated with chronic cyclic pain, dyspareunia, dysmenorrhea and sometimes dysuria. The presence of DIE, vaginal lesions and adenomyosis is strongly associated with deep dyspareunia, although the severity of the pain is not always associated with the spread of the disease (36). Endometriosis is associated with chronic inflammation, leading to tissue injury and repair, the accumulation of excess extracellular matrix (ECM) components, scar formation and ultimately, fibrosis (36). The cause of the pain symptoms should not only take anatomic abnormalities, such as adhesions and fibrosis into consideration, but also biological mechanisms, including an imbalance of sensory and sympathetic innervation due to the abnormal secretion of a variety of mediators. Estrogen promotes the growth of nerve fibers that invade endometriotic tissue and is involved in the hyperinnervation in close proximity to various nerve plexuses or the activity of neurons throughout the central nervous system (37). Altered pelvic innervation or hyperinnervation can cause pelvic pain in endometriosis via excessive neuroinflammation and subsequent neurogenesis (36). Cyclic hemorrhage from endometriosis causes local platelet activation, producing nerve growth factor (NGF, a member of the neurotrophin family) $(38,39)$. Subsequently, the sensory nerves are activated and induce neuroinflammation and excess fibrosis around endometriotic foci $(38,39)$. Furthermore, M2 macrophages infiltrating endometrial lesions produce Th2 cytokines and mediate the process of immunosuppression and neuroangiogenesis, causing the abnormal distribution of nerve fibers, which is involved in the generation of endometriosis-associated pain $(36,40)$. Since these nerve growth factors and Th2 cytokines may contribute to sensory nerve hyperinnervation, it is necessary to measure these factors in peripheral blood to objectively predict the severity of pain symptoms (41). The signaling interactions of pain mediators are described in chapter 9 below entitled 'Endometriosis-associated fibrosis: An update on molecular aspects'.

\section{Endometriosis-associated infertility: An update on clinical aspects}

The possible causes of infertility include the age of the woman, oocyte quality, sperm quality, ovarian reserve, tubal patency, uterine receptivity, anatomic abnormalities, inflammatory changes in the uterus and pelvis, and the presence of endometriosis $(15,42-45)$. Among these, endometriosis has a tremendous impact on fertility. Similar to the mechanism of pain, infertility caused by endometriosis includes anatomic and functional abnormalities.

The most likely anatomic features are pelvic adhesions and fibrosis. Adnexal adhesions are considered to impair gamete and embryo transport, resulting in reduced fertility. However, infertility cannot be explained solely by anatomic abnormalities (21). Microenvironments, such as inflammation and oxidative stress induced by endometriosis lead to ovarian dysfunction (21). Ovarian endometrioma does not adversely affect the rate of spontaneous ovulation (46). However, Liu $e t$ al reported that oocytes retrieved from women affected by endometriosis exhibited low fertilization rates due to low rates of mature oocytes (47). A meta-analysis of 27 observational studies, including 8,984 women, demonstrated that even patients with early-stage endometriosis had reduced fertilization rates compared to women without endometriosis [relative risk $(\mathrm{RR})=0.93, \mathrm{P}=0.03)]$ (48). The presence of endometriosis can cause inflammation, hormonal imbalance and oxidative stress in the eutopic endometrium, resulting in impaired endometriotic receptivity and implantation failure (49). In summary, endometriosis may affect non-ART-induced pregnancies through anatomic abnormalities of the fallopian tubes, the exposure of the endometrium to chronic inflammation, and poor egg and sperm quality. Both the ASRM committee opinion in 2012 and the European Society of Human Reproduction and Embryology (ESHRE) guideline in 2014 announced that both ovarian endometrioma per se or cystectomy for endometriomas significantly reduced the ovarian reserve and that surgical interventions for endometrioma prior to IVF treatment did not increase pregnancy rates $(1,50)$. Pal et al reported that the outcome of IVF-embryo transfer (ET) did not depend on the severity of endometriosis, suggesting that IVF-ET treatment overcomes the adverse effects on damaged oocytes (51). However, a recent meta-analysis of IVF treatment revealed that women with advanced stages of endometriosis had lower implantation and clinical pregnancy rates than patients without endometriosis ( $\mathrm{RR}=0.79, \mathrm{P}=0.0008)$ (48). Further, De Wilde et al reported that the presence of endometriosis, even in mild cases, adversely affects pregnancy outcome following IVF-ET treatment (21). Researchers have discussed that changes in the endometriosis microenvironment due to local inflammation and oxidative stress can lead to the reduced quality of the oocyte, sperm and embryo, the impaired receptivity of the endometrium and implantation failure, which has a negative effect on pregnancy rates even following IVF treatment $(15,42-45,49)$. 


\section{Endometriosis-associated infertility: An update on molecular aspects}

This chapter highlights the possible molecular mechanisms underlying infertility associated with endometriosis. Several elegant reviews have been published on the mechanisms of endometriosis-related infertility $(4,15)$. The altered expression or abnormal activation of biochemical, endocrine, immune, genetic and epigenetic factors in the endometriosis microenvironment can lead to ovarian dysfunction and subsequently, to infertility. In particular, the proinflammatory mediators (cytokines, interleukins and immune dysfunction), oxidative stress markers (hemoglobin, heme, free iron, ROS and antioxidants), hormonal imbalance, proteolytic enzymes and soluble adhesion molecules all may be potential markers for predicting endometriosis-related infertility (15). The understanding of the mechanisms through which endometriosis causes infertility may lead to the discovery of markers that predict the association between the severity of endometriosis and infertility. An overview of each of the potential markers studied will be outlined.

Inflammatory cytokines. Endometriosis is considered to be a chronic inflammatory disease $(4,52)$. Miller et al performed a comprehensive literature review of inflammatory and immune dysfunction on endometriosis-associated infertility (49). In fact, the levels of proinflammatory cytokines, including tumor necrosis factor- $\alpha$ (TNF- $\alpha$ ), interleukin (IL)- 6 and IL-8, were increased in the peritoneal fluid of women with endometriosis (49). These proinflammatory cytokines produced by activated macrophages and natural killer (NK) cells may be candidate markers for the diagnosis of infertility (53). Changes in inflammatory factors in peritoneal fluid negatively affect the fallopian tube and intrauterine environment, as the ampulla of the fallopian tube is structurally exposed to peritoneal fluid (4). These cytokines in peritoneal fluids enter the uterine cavity and increase prostaglandin production by endometrial epithelial cells, which in turn stimulates the overexpression of other inflammatory cytokines (54). Furthermore, TNF- $\alpha$, IL-6 and IL-8 activate the inflammatory response, induce angiogenesis and are also involved in tissue damage and repair. Physiological levels of inflammatory cytokines are essential for implantation, placentation, and pregnancy. The overexpression of TNF- $\alpha$, IL-6 and IL-8 can impair follicular steroidogenesis, growth and ovulation, cause impaired fertility and implantation failure, and adversely affect spontaneous pregnancy (4,54-56). Several researchers have reported that elevated serum IL-6 and IL-8 levels are associated with the occurrence of infertility (4,54-56). However, to date, to the best of our knowledge, no blood markers have been reported to determine the severity of infertility. Even other candidate markers overexpressed in the peritoneal cavity may not be reflected in the peripheral blood possibly due to dilution. Further studies are required to determine whether blood cytokine levels are associated with the severity of endometriosis-related infertility. Given the causes of infertility, cyclooxygenase (COX)-2 inhibitors, cytokine modulators, or hormone-suppressing therapies may hold promise for the treatment of infertility (15). Since the pathogenesis of endometriosis is the propagation of the inflammatory network by macrophages, the targeted inhibition of an activation cascade that will lead to an autoamplification of cytokine production, namely the cytokine storm, may be novel promising therapeutic strategies (57).

Iron and oxidative stress. Living things on earth have evolved to use iron, the fourth most abundant element in the Earth's crust, to transport oxygen. ROS are by-products produced by various cellular compartments, including mitochondria, during the process of consuming oxygen by aerobic organisms. The balance between ROS production and ROS elimination/antioxidant production is crucial to preventing ROS-induced adverse events. Physiological levels of ROS play important regulatory roles in the processes of folliculogenesis, oocyte maturation, endometrial cycle regulation, luteolysis, implantation, embryogenesis and pregnancy through various signaling pathways $(58,59)$. The excessive production of ROS causes detrimental effects on cells through lipid peroxidation, protein oxidation and DNA damage, which in turn negatively affects reproductive function $(2,52)$. Therefore, ROS act as important signaling molecules in physiological processes, while they also play a role in pathological processes involved in female reproductive function (58). Based on the above-mentioned facts, ROS act as a double-edged sword.

This chapter focuses on the iron-induced oxidative stress in endometriosis. Retrograde menstruation contains endometrial cells and red blood cells, and following hemolysis, hemoglobin, heme and free iron (so-called hemoglobin species) are released and accumulate in the pelvic cavity (14). In addition, within ovarian endometrioma, hemorrhage from the ectopic endometrium occurs at each menstruation and hemoglobin species accumulates in the cyst. Ovarian endometrioma contains higher levels of hemoglobin species compared to other types of ovarian cysts (60). Iron is a well-known inducer of oxidative stress $(14,52)$. The mechanisms through which free iron induces ROS are as follows: Iron ions react with $\mathrm{H}_{2} \mathrm{O}_{2}$ via the Fenton reaction to form hydroxyl radicals ( $\left.{ }^{\circ} \mathrm{OH}\right)$. Hydroxyl radicals are highly reactive, and therefore cause harmful oxidative damage to DNA, proteins and membrane lipids (61). The accumulation of iron in ovarian endometrioma may strongly promote an imbalance in the redox status $(61,62)$. The emerging evidence to indicate that ROS-induced oxidative stress is involved in the development and progression of endometriosis [please see the chapter entitled 'Iron-dependent progression of endometriosis' the previous study by Kobayashi et al (62)]. The comprehensive systematic review highlighted iron metabolism, oxidative stress markers (serum, peritoneal fluid, follicular fluid, ovarian cortex, and eutopic and ectopic endometrial tissue), genes involved in oxidative stress, endometriosis-induced infertility and the mechanism of carcinogenesis $(2,62)$. In addition to endometriosis, iron is also involved in cell damage, apoptosis, carcinogenesis, fibrosis, and ultimately in the dysfunction of various organs, including the heart, lungs, liver and kidneys (63).

Iron levels have been investigated in each bodily fluid of patients with endometriosis. Van Langendonckt et al reported that iron levels in the peritoneal cavity of patients with endometriosis were increased compared to the controls, although serum iron levels were not (14). However, Alizadeh et al found that serum iron levels in patients with endometriosis were significantly higher compared with the controls (64). Serum 
iron allowed for the discrimination between patients with endometriosis and controls with an area under the receiver operating characteristic (ROC) curve (AUC) of 0.899 and a cut-off value of $1.73 \mathrm{mg} / \mathrm{l}$ (64). In addition, the median \pm SD total iron, heme and free iron levels in endometriotic cyst fluids were previously shown to be $244.4 \pm 204.9,303.9 \pm 324.4$ and $13.5 \pm 16.2 \mathrm{mg} / \mathrm{l}$, respectively (60). Thus, the iron levels in ovarian endometrioma were 10-100-fold higher than those in serum, depending on the hemoglobin species. High levels of intracystic iron can oxidize a wide range of substrates and cause biological damage and cell death to adjacent follicles (64). Following the degradation of hemoglobin, iron is released from heme and accumulates in tissues over a period of several days, which results in the deposition of hemosiderin.

However, iron levels in bodily fluids of women with endometriosis remain controversial. Montoya-Estrada et al reported that the levels of peritoneal hemoglobin did not differ between the endometriosis group and the non-endometriosis group (65). Benaglia et al reported that there was no significant difference in iron concentrations in follicular fluid between ovaries affected with endometriosis and those not affected (66). These data suggest that high levels of iron in ovarian endometrioma do not affect oocyte quality as they are less likely to spread through the cyst wall. Despite conflicting data, a number of studies support the notion that iron-induced oxidative stress impairs fertility in women with endometriosis $(2,52,56,59,63,64)$.

The iron concentration that adversely affects fertility is unknown. In another study, basic research on iron-related oxidative stress was performed on cerebral hemorrhage. In animal models, recovery, injury, sequelae, or death from intracerebral hemorrhage may depend on the degree of hemolysis, iron release and oxidative stress that occurs following hemorrhage (67). Therefore, iron chelators may prevent neuronal death induced by oxidative stress. Experiments using Medaka fish have demonstrated that oxidation products (e.g., $\mathrm{Fe}^{3+}$ ) at lower $\mathrm{mg} / \mathrm{l}$ levels can induce reproductive toxicity through oxidative stress (68). Although humans and fish cannot be compared, taking the serum iron levels $(>1.73 \mathrm{mg} / \mathrm{l})$ of women with endometriosis into consideration, the possibility of reproductive toxicity due to direct gonadal damage cannot be ruled out.

The oxidant-antioxidant imbalance. Herein, the oxidant-antioxidant balance in bodily fluids in patients with endometriosis is reviewed. Several studies have identified a variety of indicators of oxidative stress and antioxidant capacity. Some indicators of oxidative stress, such as ROS and iron, are elevated in the endometriosis group compared to other infertile women without endometriosis or tubal infertility (69). Extensive damage to DNA by hemoglobin species is reflected as an increase in 8-hydroxy-2'-deoxyguanosine (8-OHdG) levels in endometriotic cyst fluid (70) and cell nuclei (71). There is evidence suggests that women with stage I/II endometriosis have higher levels of $8-\mathrm{OHdG}$ in follicular fluid compared to the controls $(24.21 \pm 8.56$ vs. $17.22 \pm 5.6 \mathrm{ng} / \mathrm{ml})(72,73)$. Even in the early stages of endometriosis, they are exposed to oxidative stress and are associated with reduced oocyte quality (72). In addition, the levels of markers of acute oxidative stress, such as malondialdehyde (MDA), the most mutagenic product of lipid peroxidation (2), carbonyls and lipohydroperoxides (LOOH) (65), myeloperoxidase (MPO) (74), lipid peroxidation (LPO) (69) and oxidized low-density lipoprotein (oxLDL) (2) are elevated in the peripheral blood, peritoneal fluids, or follicular microenvironments in patients with endometriosis $(73,75)$. The levels of oxidative stress, represented by LOOH and MPO, are associated with the severity of endometriosis, and are inversely associated with the percentage of mature oocytes, the implantation rate and clinical pregnancy rate (8,69,74-76). These data indicate that oxidative stress exerts a major effect on oocyte, sperm, and embryo quality and function.

Contrary to the level of oxidative stress, the levels of several antioxidant molecules are significantly lower in patients with endometriosis than in those without endometriosis. Molecules associated with antioxidant capacity include a subunit of the cystine/glutamate transporter xCT (SLC7A11), glutathione peroxidase 4 (GPX4) and the glutamate-cysteine ligase (GCLC), glutathione reductase (GR), catalase, superoxide dismutase (SOD), iron metabolism genes transferrin receptor 1 (TfR1), ferroportin (Fpn), heme oxygenase 1 (HO-1) and ferritin that are regulated by the antioxidant response element of the transcription factor, nuclear factor, erythroid 2 like 2 (NRF2) $(52,69,77,78)$. Insufficient antioxidant capacity promotes harmful ROS formation (52). In addition, recent studies have focused on the antioxidants, HO-1 (79) and CD44v9 (71). HO-1 is constitutively present in M2 phenotype macrophages and exerts beneficial protective effects against oxidative damage. In addition, CD44v9 expressed in endometriotic cells stabilizes the glutamate-cystine transporter $\mathrm{xCT}$, thereby decreasing the intracellular levels of ROS (71). The CD44v9 system is involved in survival, anti-apoptosis and anti-oxidative stress by maintaining higher levels of antioxidants (80). In an oxidative stress environment, iron-dependent lipid peroxidation leads to regulated non-apoptotic cell death, also known as ferroptosis $(78,81)$. Endometriosis is characterized by resistance to ferroptosis (82). In addition to endometriosis, ferroptosis has been implicated in the pathological cell death associated with degenerative diseases, stroke, intracerebral hemorrhage, traumatic brain injury, ischemia-reperfusion injury, kidney degeneration and carcinogenesis (81). It is necessary to elucidate the detailed mechanisms of avoiding ferroptosis in endometriosis with reference to the molecular mechanisms of these diseases.

It would also be of interest to elucidate the mechanisms through which the oxidant-antioxidant imbalance adversely affects fertility. There are considerable basic and clinical studies supporting that ROS affects human fertility. Notarstefano et al reported that the oxidative stress-induced dysregulation of lipid and carbohydrate metabolism occurs in the ovary affected by endometriotic lesions, as well as in the contralateral healthy ovary (83). This cannot be explained by the direct action of endometriosis alone, but by biological, biochemical, genetic, or epigenetic modifications which may occur in pelvic organs possibly through ROS-induced oxidative stress. It has been demonstrated that the redox balance also affects the clinical performance of ART. Follicular fluid 8-OHdG levels have been shown to be inversely associated with the fertilization rate following intra-cytoplasmic sperm injection (ICSI) (84). In addition, the levels of follicular fluid and seminal plasma MDA are inversely associated with the IVF results (85). On the 
other hand, follicular fluid total GSH levels, total antioxidant capacity (TAC) and total systemic antioxidant response (TAR) are positively associated with the fertilization rate following ICSI (86). Previously, a positive association was observed between the increased expression of SOD1 in cumulus cells and the clinical pregnancy rate (87). Given that patients with endometriosis have a lower follicular total GSH activity and that IVF-ET/ICSI is successful in patients with endometriosis with a high blood antioxidant capacity, it is likely that the oxidant-antioxidant balance has a significant effect on fertility (86). Since ROS in the culture medium are known to adversely affect the post-fertilization cleavage rate and blastocyst yield and quality (76), the supplementation of antioxidants to the medium may increase the success rate of ART through the reduction of oxidative stress (13). The degree of oxidative stress in follicular fluid, peritoneal fluid and peripheral blood may be involved in infertility by regulating the expression of genes encoding cytokines and immunomodulators that are associated with sustained inflammatory response and immune dysregulation (14). Taken together, these findings strongly suggest that changes in the microenvironment due to oxidative stress contribute to infertility.

Apart from endometriosis, the oxidant-antioxidant imbalance has been described in several pathophysiological conditions, including reproductive disorders, such as polycystic ovary syndrome (PCOS) and infertility of unknown origin (13). In addition, being overweight, smoking and alcohol consumption may affect fertility by promoting excessive amounts of free radical production (13). Therefore, it is well known that a healthy lifestyle can maintain good fertility.

Conversely, certain studies have demonstrated opposite results, indicating that oxidative stress does not affect reproductive function. The total oxidant status and antioxidant capacity in the follicular fluid of patients with unilateral ovarian endometrioma have been shown to not differ from those without endometrioma (88). Furthermore, oxidative stress in follicular fluid has been shown to not impair oocyte fertility (89). The association between oxidative stress and fertility remains controversial; however, a number of studies have demonstrated increased oxidative stress and reduced fertility in patients with endometriosis $(2,52,58,64)$. There are at least two possible mechanisms for endometriosis-related infertility: First, excessive oxidative stress in follicular fluid leads to reduced antioxidant capacity, and second, low antioxidant capacity results in high levels of oxidative stress.

If oxidative stress is really involved in the development of endometriosis, available antioxidants or iron chelators can be selected as novel therapeutic targets. In preliminary experiments using animals, antioxidants may be possible therapeutic candidates for endometriosis-related fibrosis, which are lipophilic antioxidants, including vitamin E, polyphenols, ferrostatin-1 (Fer-1), or liproxstatin-1 (Lip-1) (90). Epigallocatechin-3-gallate (EGCG) suppresses endometriosis-associated fibrosis by inhibiting the transforming growth factor-1 $\beta$ (TGF- $\beta 1$ )-stimulated activation of Smad and mitogen-activated protein kinase (MAPK) signaling pathways (91). The injection of an iron chelator, deferoxamine, into a mouse model of endometriosis suppressed the growth of endometriotic lesions (92). Certain antioxidants, such as EGCG and deferoxamine, are effective in the treatment of endometriosis and fibrosis. There are only a few animal studies to identify effective therapeutic candidates for endometriosis $(91,92)$. Showell et al reviewed whether oral antioxidant supplements improve fertility in infertile women (93). Limited evidence suggests that antioxidants, such as $\mathrm{N}$-acetyl-cysteine, melatonin, L-arginine, carnitine, selenium, vitamin E, vitamin B, vitamin C, vitamin D and CoQ10, improve fertility (93). To date, to the best of our knowledge, no clinical studies have been conducted using available antioxidant supplements exclusively in infertile women with endometriosis.

Iron-dependent progression of endometriosis. It would be of interest to determine the reasons why endometriosis requires toxic iron for its development and progression. The authors have previously summarized the recent advances in our understanding of the underlying epigenetic mechanisms focusing on oxidative stress in endometriosis (94). The hypothesis is as follows: Iron modulates the expression of genes involved in methylation, creating an environment that contributes to impaired decidualization and favors the growth of endometriotic cells (94). Iron-induced oxidative stress alters the activity of enzymes responsible for the demethylation and deacetylation of histones (95) and regulates the expression of CpG demethylases, such as ten-eleven translocation (TET) and jumonji (JMJ) (94). These demethylases recognize a wide range of endogenous DNA methylation (94). The steroid hormone-mediated decidual signaling pathway has been shown to be dysregulated in endometriosis through the modification of DNA methylation. DNA methylation is also closely involved in epithelial-mesenchymal transition (EMT), such as the suppression of E-cadherin, and the increased expression of vascular endothelial growth factor (VEGF) (96), which may contribute to the development and progression of endometriosis. There are at least 2 distinct phases of epigenetic modification in endometriosis: The initial wave of iron-induced oxidative stress would be followed by the second big wave of epigenetic modulation of endometriosis susceptibility genes (94). Taken together, endometrial and endometriotic foci with localized iron overload are important to the subsequent pathophysiology of the development of endometriosis. Oxidative stress is required for endometriosis to survive and, as the lesion progresses, it causes symptoms, such as pain and infertility.

\section{Endometriosis-associated fibrosis: An update on molecular aspects}

It is generally considered that the greater the number of anatomical distortions due to endometriosis adhesions and fibrosis, the more often infertility occurs. Furthermore, soluble factors such as inflammation, oxidative stress, endocrine abnormalities and immunological disturbances are also important causes of infertility in women with endometriosis (15,42-45). Chronic wounds, such as not only endometriosis, but also diabetic foot wounds and ulcers, usually deteriorate into non-healing wounds, undergoing repeated tissue injury and repair $(40,97,98)$. Endometriosis-related fibrosis is triggered by an inflammatory response, causing EMT, fibroblast-myofibroblast transdifferentiation (FMT) and smooth muscle metaplasia (SMM), and repeating this cycle $(4,40,97,98)$. Guo et al found that older endometriotic cysts had higher concentrations of 


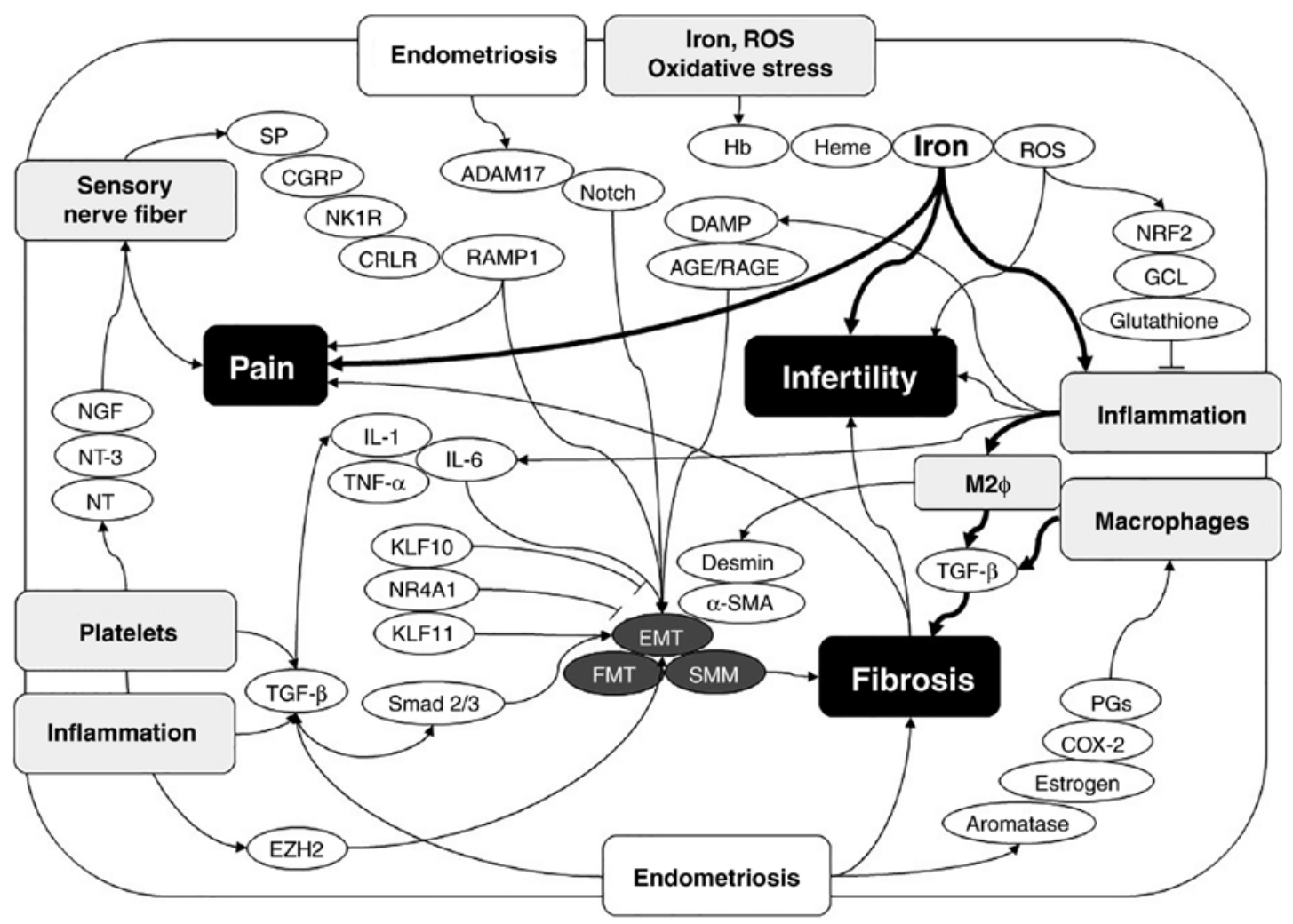

Figure 2. Molecular mechanisms underlying the regulation of endometriosis-associated pain, infertility and fibrosis. A variety of symptoms of endometriosis, such as pain, infertility and fibrosis, involve interrelated signaling mechanisms. First, endometriosis-associated pain symptoms are caused by humoral factors from inflammation and activated platelets, resulting in sensory nerve hyperinnervation. Second, infertility may be affected by oxidative stress, inflammation and finally fibrosis. Finally, fibrosis is formed by the interaction of inflammation, M2 macrophages, activated platelets, sensory nerve hyperinnervation, oxidative stress and iron. ADAM17, a disintegrin and metalloproteinase-17; $\alpha$-SMA, $\alpha$-smooth muscle actin; CGRP, calcitonin gene-related peptide; COX-2, cyclooxygenase-2; CRLR, calcitonin receptor like receptor; EZH2, enhancer of zeste 2 polycomb repressive complex 2 subunit; GCL, glutamate-cysteine ligase; Hb, hemoglobin; IL-1, interleukin-1; IL-6, interleukin-6; KLF10, Kruppel like factor 10; KLF11, Kruppel like factor 11; NGF, nerve growth factor; NK1R, neurokinin 1 receptor; NR4A1, nuclear receptor subfamily 4 group A member 1; NRF2, nuclear factor, erythroid 2 like 2; NT, neurotrophin; NT-3, neurotrophin-3; PGs, prostaglandins; SP, substance P; RAMP1, receptor activity modifying protein 1 ; ROS, reactive oxygen species; TGF- $\beta$, transforming growth factor- $\beta$; and TNF- $\alpha$, tumor necrosis factor- $\alpha$.

total bilirubin, ferritin, free iron and collagen, exhibited higher density and viscosity, and also exhibited stronger adhesions than younger cysts (97). Over time, ROS from accumulated iron promote fibrosis, forming strong adhesions around endometriotic lesions and eventually replacing the ovaries with fibrous tissue (99). Fibrosis also physically causes vascular damage, which can result in follicular loss, reduced ovarian reserve and infertility $(4,99,100)$.

The major cell types involved in the development of endometriosis-associated fibrosis are platelets, various inflammatory cells, such as macrophages, T lymphocytes, B lymphocytes and NK cells, ectopic endometrial cells and sensory nerve fibers $(101,102)$. These cells produce key effectors. such as fibrosis-related cytokines and chemokines (TGF- $\beta$, TNF- $\alpha$, IL-1 and L-6), a variety of neuropeptides, injury-related molecular patterns, such as damage-associated molecular pattern (DAMP) and receptor for advanced glycation end-products (RAGE) (101,102). This chapter describes the molecular mechanisms involved in endometriosis fibrosis on a cell-by-cell basis. The molecular mechanisms that regulate endometriosis-associated pain, infertility and fibrosis are illustrated in Fig. 2.

Platelets. Platelets are involved in endometriosis-associated fibrosis, immune regulation and hyperinnervation. TGF- $\beta 1$ produced by activated platelets promotes fibrosis through the TGF- $\beta /$ Smad signaling pathway in endometriosis (103). Furthermore, endometrial stromal cells, upon exposure to activated platelets, induce alpha-smooth muscle actin (SMA) expression and promote fibrosis (103). These results indicate that neurotrophic factors produced by activated platelets around the lesions play an important role in endometriosis-related fibrosis $(38,103)$. Activated platelets are also involved in organ fibrosis in a number of diseases other than endometriosis. Platelet-derived TGF- $\beta 1$ stimulates pathological fibrosis in a variety of important organs such as the heart, liver, lungs and kidneys (104). Furthermore, platelet-derived TGF- $\beta 1$ induces the reduced cytotoxicity of NK cells in patients with endometriosis, suggesting that platelets may directly participate in the progression of endometriosis as immune-like cells (105). Finally, human platelets are rich in neurotrophic factors, which consist of nerve grow th factor (NGF), brain-derived neurotrophic factor (BDNF), and neurotrophin 3 (NT-3) and neurotrophin 4/5 (NT4/5) (39). The members of the neurotrophin family are essential for the development, maturation, maintenance and homeostasis of the nervous system, particularly by regulating cell differentiation, survival, synaptic growth and death (39). Endometriotic lesions and surrounding tissues can be hyperinnervated, possibly by NTs secreted by platelets (38). Furthermore, the neurotrophic 
factors induce the differentiation of sensory neurons, which are involved in all major steps in the wound healing and fibrosis of endometriotic lesions (38). Substance P (SP), also referred to as neurokinin 1 (NK1), induces EMT, FMT, SMM and cell contractility, produces collagen and matrix proteins, exacerbates fibrosis and hyperalgesia, and increases lesion weight via neurokinin 1 receptor (NK1R) activation $(38,41)$. Therefore, SP and its receptor, NK1R, may be candidate molecular targets for the treatment of fibrosis.

Macrophages. In response to tissue damage, a variety of inflammatory cells (macrophages and monocytes) and other cells (hematopoietic, stromal and stem cells) are recruited to tissue injury sites where they promote changes in the microenvironment (98). Among the inflammatory cell types, tissue macrophages and inflammatory monocytes are involved in all processes of initiation, maintenance and the resolution of inflammation $(40,106)$. Macrophages adapt to environmental changes to alter proinflammatory (M1) and anti-inflammatory (M2) phenotypes and functions (106). High ROS levels induced by iron overload promote macrophages to polarize into M1-type macrophages (107). On the other hand, sustained increases in iron levels lead to polarization toward the type 2 response (108). Macrophages polarize over time toward the M2 subtype to reduce inflammation and tissue destruction by M1 macrophages. Research using experimental animal models has demonstrated that M2 macrophages induce EMT, FMT and SMM to promote endometriosis-associated fibrosis (40). Iron establishes a vicious cycle through the activation of macrophages, changes in polarity, increased oxidative stress and enhanced fibrosis $(59,109)$. Since fibrosis or scarring due to persistent inflammation impairs ovarian function, M1/M2 macrophages may represent a novel therapeutic target (98).

Sensory nerve fibers. Among the various phenotypes of endometriosis, DIE in particular is known to be hyperinnervated due to its proximity to the pelvic plexus (41). Compared with ovarian endometrioma, several neuropeptide hormones are overexpressed in DIE, resulting in higher nerve fiber density (41). Neuropeptides such as SP, NK1R, calcitonin receptor like receptor (CRLR), receptor activity modifying protein 1(RAMP-1) and calcitonin gene-related peptide (CGRP) are likely to play a role in the bidirectional communication both within the nervous system and between neurons and endometriotic cells (41). The above-mentioned neuropeptide hormones induce the expression of genes related to EMT and fibrosis, such as $\alpha$-SMA, desmin, oxytocin receptor and smooth muscle myosin heavy chain (41). The severity of DIE is associated with the expression levels of neuropeptides, suggesting that sensory nerves play a significant role in endometriosis-associated pain and fibrosis (41). Furthermore, neuropeptide hormones, such as NGF and NT-3 secreted from peritoneal lesions promote the growth of nerve fibers in and around peritoneal endometriotic lesions, resulting in adhesions and pain (110).

Candidate markers for fibrotic endometriosis. This chapter summaries individual markers strongly associated with endometriosis-related fibrosis.
$T G F-\beta 1$. Peritoneal mesothelial cells are damaged in the inflammatory process, which activates pro-inflammatory mediators, including peritoneal cytokines (TGF- $\alpha$, TGF- $\beta$, TNF- $\alpha$, IL-1, IL- 6 and prostaglandins), produces growth factors (VEGF, epidermal growth factor and platelet-derived growth factor) and promotes the fibrin/coagulation cascade [thrombin, tissue factor, plasminogen activator inhibitor (PAI)-1/2], leading to tissue healing and repair (111). Among these mediators, TGF- $\beta 1$ is predominantly expressed by macrophages, peritoneal mesothelial cells and endometriotic cells, and promotes cell growth, migration, extracellular matrix production, adhesions and fibrosis possibly through the TGF- $\beta 1 / \mathrm{Smad}$ and TGF- $\beta 1 /$ hypoxia-inducible factor-1 (HIF-1) signaling pathways $(103,111)$. Furthermore, TGF- $\beta 1$ strongly induces peritoneal mesothelial-mesenchymal transition, adhesion and ultimately fibrosis formation in the presence of inflammatory cytokine such as IL-6 $(103,111)$. Thus, TGF- $\beta$ is considered to be the most important factor in endometriosis-related fibrosis (100).

Nuclear receptor subfamily 4 group A member 1 (NR4A1). Zeng et al found that the nuclear transcription factor, NR4A1, reduced fibrosis through the suppression of the TGF- $\beta / \mathrm{Smad}$ signaling pathway (100). In in vitro studies, as well as in animal experiments, NR4A1 suppressed the progression of fibrosis though the downregulation of collagen type I alpha 1 chain (COL1A1) expression (100). NR4A1 may thus serve as the molecular target and therapy for the management of fibrosis (100).

Kruppel like factor 11 (KLF11). The transcription factor, KLF11, binds to the promoter regions of collagen, matrix metalloproteinases and TGF- $\beta$ family genes in endometrial stromal cells and suppresses their protein expression, resulting in inhibition of fibrosis formation (112). In an animal model of endometriosis, Klf11 $1 /$ animals were shown to develop multiple fibrous adhesions (112). On the other hand, K1f10, a paralog of Klf11, exerts the opposite effect of Klf11 and promotes fibrosis (113).

Aromatase. Endometrial cells that overexpress aromatase protect themselves from destruction by activated macrophages via estrogen production (114). Keskin et al found that the aromatase inhibitor reduced postoperative adhesion formation in the rat uterine horn experimental model (115). Maia et al demonstrated that aromatase was overexpressed in endometriotic cells through an epigenetic alteration that stimulated the demethylation of the promoter region (114). On the other hand, however, enhanced estrogen production by aromatase overexpression promotes prostaglandin synthesis by stimulating COX-2 activity (114). In addition, prostaglandin is involved in tissue remodeling by promoting angiogenesis and fibrosis (114). Although the molecular mechanisms of adhesion and fibrosis may differ, the effect of aromatase on fibrosis remains controversial.

Enhancer of zeste 2 polycomb repressive complex 2 subunit (EZH2).EZH2 is a subunit of the polycomb repressive complex 2 (PRC2) catalyzing the trimethylation of histone $\mathrm{H} 3$ lysine 27 (H3K27) (116). Xiao et al found that EZH2 induced angiogenesis, EMT, myofibroblast transformation and tissue fibrosis through the TGF $\beta /$ Smad signaling pathway and the upregulation of vimentin and transcription factors, such as Snail and Slug, and the downregulation of E-cadherin (116). Zhang et al found that $\mathrm{EZH} 2$ was elevated not only in the ectopic endometrium of 
endometriosis, but also in the eutopic endometrium compared to the control endometrium (117). In fact, an EZH2 inhibitor, 3-deazaneplanocin A (DZNep), inhibited cell growth, EMT and fibrosis in a preclinical mouse model of endometriosis (117). Activated platelets also upregulate EZH2 expression, leading to endometrial invasion and fibrosis (117). EZH2 is an important epigenetic regulator as a marker of endometriosis-associated fibrosis and may prove to be a potential therapeutic candidate for the suppression of fibrosis.

$N R F 2$. NRF2, a downstream target of the Kelch-like $\mathrm{ECH}$-associated protein (KEAP1), upregulates anti-inflammatory gene expression and inhibits the progression of inflammation via the antioxidant response element (ARE) signaling pathway (118). Marcellin et al found that NRF2 expression in ectopic endometrial tissue was decreased compared to the eutopic endometrium in women with endometriosis and without disease (119). The reduced NRF2 gene expression may suppress the transcription of endogenous antioxidants, such as glutathione and activate oxidative damage and inflammatory response, thereby promoting the progression and fibrosis of endometriosis (119). Furthermore, a study using a mouse model of endometriosis revealed that transplantation of NRF2 ${ }^{-/-}$cells was more fibrotic compared to wild-type cells (119). These data suggest that NRF2 may be a potential candidate for the suppression of fibrotic endometriosis.

A disintegrin and metalloproteinase-17 (ADAM17). Tissue damage upregulates the expression of ADAM17, promotes macrophages and neutrophil infiltration, and eventually causes further fibrosis (120). González-Foruria et al found that oxidative stress in endometriosis caused fibrosis via the ADAM17/Notch signaling pathway (12). The Notch pathway is involved in the regulation of myofibroblast differentiation in chronic fibrosis including in the lung, kidney, liver, heart and skin (121). Therefore, the ADAM17/Notch signaling pathway is essential for fibrosis in diverse organs and tissues (121).

Lysyl oxidase (LOX). LOX plays crucial roles in tissue remodeling and fibrosis due to its crosslinking of soluble collagen and elastin into insoluble, mature fibers (122). Elevated LOX levels catalyze collagen crosslinking to induce irreversible chronic fibrosis in a variety of organs, including the kidneys. LOX is also involved in the progression of endometriosis by promoting cell invasion, EMT and fibrosis (122).

Others. In addition to the mediators mentioned above, endometriosis-associated fibrosis also involves damage-associated molecular patterns (DAMP), pattern recognition receptors, advanced glycation end products (AGE), receptor for advanced glycation end products (RAGE), extracellular lactate and increased glycolysis (111). The activation of pattern recognition receptor by DAMP stimulates cells of the innate immune system to further activate toxic inflammatory responses, promoting myofibroblast activation, fibrosis, or cell death (123). High-mobility group box-1 (HMGB1), a member of the DAMP family, increases in response to tissue damage infection, inflammation, immune responses and fibrosis by binding to specific cell-surface receptors (124). The expression of RAGE (125) and its ligand, HMGB1 (124), is increased in endometriosis. Cao et al reported that HMGB1 participated in the pathogenesis of endometriosis-associated fibrosis though RAGE signaling mechanisms (124).

\section{Conclusion and future perspectives}

Currently, the assessment of disease severity for endometriosis is based on anatomical findings; however, no single classification or scoring system has proven satisfactory. In order to solve this issue and create a disease severity score tailored to each symptom of endometriosis, not only anatomical findings, but also biochemical parameters that objectively reflect the severity of the disease are required. Oxidative stress and inflammation serve as common biomarkers for the characteristic symptoms of endometriosis, as illustrated by the bold curved arrows in Fig. 2. Among these biomarkers, the level of iron in peritoneal fluid, ovarian endometrioma and peripheral blood may reflect the severity of the disease. Future studies on easily available biomarkers will provide useful information for the development of novel classification systems for endometriosis. Both hormonal therapy and surgical resection are currently the main treatments for endometriosis; however, the elucidation of etiology and pathophysiology will provide non-hormonal treatments to women suffering from severe pain and women who wish to become pregnant (126). The management of endometriosis should be individualized, considering the patient's background, risk factors and disease severity (16). The present review may prove to be useful for exploring available biomarkers that are associated with endometriosis-specific disease severity such as pain, infertility and fibrosis.

\section{Acknowledgements}

Not applicable.

\section{Funding}

The present study was supported by the Japan Society for the Promotion of Science (JSPS) Grants-in-Aid for Scientific Research (KAKENHI) (grant nos. JP16K11150, 18K09269 and $18 \mathrm{~K} 09234$ to HK).

\section{Availability of data and materials}

Not applicable.

\section{Author's contributions}

SI, SM, MK and MN performed the literature search and collected data using web-based databases. SI and HK made substantial contribution to conception of the study. SI contributed to the study design and interpretation of the included research studies. The final version of the manuscript has been read and approved by all authors.

\section{Ethics approval and consent to participate}

Not applicable.

\section{Patient consent for publication}

Not applicable. 


\section{Competing interests}

The authors declare that they have no competing interests.

\section{References}

1. Dunselman GA, Vermeulen N, Becker C, Calhaz-Jorge C, D'Hooghe T, De Bie B, Heikinheimo O, Horne AW, Kiesel L, Nap A, et al: ESHRE guideline: Management of women with endometriosis. Hum Reprod 29: 400-412, 2014.

2. Scutiero G, Iannone P, Bernardi G, Bonaccorsi G, Spadaro S, Volta CA, Greco P and Nappi L: Oxidative stress and endometriosis: A systematic review of the literature. Oxid Med Cell Longev 2017: 7265238, 2017.

3. Adamson GD: Endometriosis classification: An update. Curr Opin Obstet Gynecol 23: 213-220, 2011

4. Donnez J, Donnez O, Orellana R, Binda MM and Dolmans MM: Endometriosis and infertility. Panminerva Med 58: 143-150, 2016.

5. Spaczynski RZ and Duleba AJ: Diagnosis of endometriosis Semin Reprod Med 21: 193-208, 2003.

6. Guerriero S, Condous G, van den Bosch T, Valentin L, Leone FP, Van Schoubroeck D, Exacoustos C, Installé AJ, Martins WP, Abrao MS, et al: Systematic approach to sonographic evaluation of the pelvis in women with suspected endometriosis, including terms, definitions and measurements: A consensus opinion from the international deep endometriosis analysis (IDEA) group. Ultrasound Obstet Gynecol 48: 318-332, 2016.

7. Reid S and Condous G: Update on the ultrasound diagnosis of deep pelvic endometriosis. Eur J Obstet Gynecol Reprod Biol 209: 50-54, 2017.

8. Da Broi MG and Navarro PA: Oxidative stress and oocyte quality: Ethiopathogenic mechanisms of minimal/mild endometriosis-related infertility. Cell Tissue Res 364: 1-7, 2016.

9. Sanchez AM, Vanni VS, Bartiromo L, Papaleo E, Zilberberg E, Candiani M, Orvieto $\mathrm{R}$ and Viganò $\mathrm{P}$ : Is the oocyte quality affected by endometriosis? A review of the literature. J Ovarian Res 10: 43, 2017.

10. Laganà AS, Garzon S, Götte M, Viganò $\mathrm{P}$, Franchi M, Ghezzi $\mathrm{F}$ and Martin DC: The pathogenesis of endometriosis: Molecular and cell biology insights. Int J Mol Sci 20: pii: E5615, 2019.

11. Rolla E: Endometriosis: Advances and controversies in classification, pathogenesis, diagnosis, and treatment. F1000Res 8: pii: F1000 Faculty Rev-529, 2019.

12. González-Foruria I, Santulli P, Chouzenoux S, Carmona F, Chapron C and Batteux F: Dysregulation of the ADAM17/Notch signalling pathways in endometriosis: From oxidative stress to fibrosis. Mol Hum Reprod 23: 488-499, 2017.

13. Agarwal A, Aponte-Mellado A, Premkumar BJ, Shaman A and Gupta S: The effects of oxidative stress on female reproduction: A review. Reprod Biol Endocrinol 10: 49, 2012.

14. Van Langendonckt A, Casanas-Roux F and Donnez J: Oxidative stress and peritoneal endometriosis. Fertil Steril 77: 861-870, 2002.

15. Gupta S, Goldberg JM, Aziz N, Goldberg E, Krajcir N and Agarwal A: Pathogenic mechanisms in endometriosis-associated infertility. Fertil Steril 90: 247-257, 2008.

16. Chapron C, Marcellin L, Borghese B and Santulli P: Rethinking mechanisms, diagnosis and management of endometriosis. Nat Rev Endocrinol 15: 666-682, 2019.

17. Holland TK, Yazbek J, Cutner A, Saridogan E, Hoo WL and Jurkovic D: Value of transvaginal ultrasound in assessing severity of pelvic endometriosis. Ultrasound Obstet Gynecol 36: 241-248, 2010.

18. Zanardi R, Del Frate C, Zuiani C and Bazzocchi M: Staging of pelvic endometriosis based on MRI findings versus laparoscopic classification according to the American fertility society. Abdom Imaging 28: 733-742, 2003.

19. Guerriero S, Ajossa S, Garau N, Alcazar JL, Mais V and Melis GB: Diagnosis of pelvic adhesions in patients with endometrioma: The role of transvaginal ultrasonography. Fertil Steril 94: 742-746, 2010.

20. Gerges B, Lu C, Reid S, Chou D, Chang T and Condous G: Sonographic evaluation of immobility of normal and endometriotic ovary in detection of deep endometriosis. Ultrasound Obstet Gynecol 49: 793-798, 2017.
21. De Wilde RL, Alvarez J, Brölmann H, Campo R, Cheong Y, Lundorff P, Pawelczyk L, Roman H, di Spiezio Sardo A and Wallwiener M: Adhesions and endometriosis: Challenges in subfertility management: (An expert opinion of the ANGEL-the anti-adhesions in gynaecology expert panel-group). Arch Gynecol Obstet 294: 299-301, 2016.

22. Ichikawa M, Akira S, Kaseki H, Watanabe $\mathrm{K}$, Ono $\mathrm{S}$ and Takeshita T: Accuracy and clinical value of an adhesion scoring system: A preoperative diagnostic method using transvaginal ultrasonography for endometriotic adhesion. J Obstet Gynaecol Res 46: 466-478, 2020

23. Haas D, Shebl O, Shamiyeh A and Oppelt P: The rASRM score and the Enzian classification for endometriosis: Their strengths and weaknesses. Acta Obstet Gynecol Scand 92: 3-7, 2013.

24. Tuttlies F, Keckstein J, Ulrich U, Possover M, Schweppe KW, Wustlich M, Buchweitz O, Greb R, Kandolf O, Mangold R, et al: ENZIAN-score, a classification of deep infiltrating endometriosis. Zentralbl Gynakol 127: 275-281, 2005.

25. Morgan-Ortiz F, López-de la Torre MA, López-Zepeda MA, Morgan-RuizFV,Ortiz-BojórquezJC and Bolívar-Rodríguez MA: Clinical characteristics and location of lesions in patients with deep infiltrating endometriosis using the revised Enzian classification. J Turk Ger Gynecol Assoc 20: 133-137, 2019.

26. Haas D, Wurm P, Shamiyeh A, Shebl O, Chvatal R and Oppelt P: Efficacy of the revised Enzian classification: A retrospective analysis. Does the revised Enzian classification solve the problem of duplicate classification in rASRM and Enzian? Arch Gynecol Obstet 287: 941-945, 2013.

27. Endometriosis stages: Understanding the different stages of endometriosis (https://www.endofound.org/endometriosis-stages).

28. Adamson GD and Pasta DJ: Endometriosis fertility index: The new, validated endometriosis staging system. Fertil Steril 94: 1609-1615, 2010

29. Andres MP, Borrelli GM and Abrão MS: Endometriosis classification according to pain symptoms: Can the ASRM classification be improved? Best Pract Res Clin Obstet Gynaecol 51: 111-118, 2018.

30. Revised American society for reproductive medicine classification of endometriosis: 1996. Fertil Steril 67: 817-821, 1997.

31. Sapkota Y, Attia J, Gordon SD, Henders AK, Holliday EG, Rahmioglu N, MacGregor S, Martin NG, McEvoy M, Morris AP, et al: Genetic burden associated with varying degrees of disease severity in endometriosis. Mol Hum Reprod 21: 594-602, 2015.

32. Stovall DW, Bowser LM, Archer DF and Guzick DS Endometriosis-associated pelvic pain: Evidence for an association between the stage of disease and a history of chronic pelvic pain. Fertil Steril 68: 13-18, 1997.

33. Pop-Trajkovic S, Popović J, Antić V, Radović D, Stefanović M and Vukomanović P: Stages of endometriosis: Does it affect in vitro fertilization outcome. Taiwan J Obstet Gynecol 53: 224-226, 2014

34. Johnson NP, HummelshojL, Adamson GD, Keckstein J, TaylorHS Abrao MS, Bush D, Kiesel L, Tamimi R, Sharpe-Timms K, et al: World Endometriosis Society consensus on the classification of endometriosis. Hum Reprod 32: 315-324, 2017.

35. Haas D, Oppelt P, Shebl O, Shamiyeh A, Schimetta W and Mayer R: Enzian classification: Does it correlate with clinical symptoms and the rASRM score? Acta Obstet Gynecol Scand 92: $562-566,2013$.

36. Wu J, Xie H, Yao S and Liang Y: Macrophage and nerve interaction in endometriosis. J Neuroinflammation 14: 53, 2017.

37. Berkley KJ, Rapkin AJ and Papka RE: The pains of endometriosis. Science 308: 1587-1589, 2005.

38. Liu X, Yan D and Guo SW: Sensory nerve-derived neuropeptides accelerate the development and fibrogenesis of endometriosis. Hum Reprod 34: 452-468, 2019.

39. Rocco ML, Soligo M, Manni L and Aloe L: Nerve growth factor: Early studies and recent clinical trials. Curr Neuropharmacol 16: $1455-1465,2018$

40. Duan J, Liu X, Wang H and Guo SW: The M2a macrophage subset may be critically involved in the fibrogenesis of endometriosis in mice. Reprod Biomed Online 37: 254-268, 2018.

41. Yan D, Liu X and Guo SW: Neuropeptides substance P and calcitonin gene related peptide accelerate the development and fibrogenesis of endometriosis. Sci Rep 9: 2698, 2019.

42. Azem F, Lessing JB, Geva E, Shahar A, Lerner-Geva L, Yovel I and Amit A: Patients with stages III and IV endometriosis have a poorer outcome of in vitro fertilization-embryo transfer than patients with tubal infertility. Fertil Steril 72: 1107-1109, 1999. 
43. Garrido N, Navarro J, García-Velasco J, Remoh J, Pellice A, Simón C: The endometrium versus embryonic quality in endometriosis-related infertility. Hum Reprod Update 8: 95-103, 2002.

44. Augoulea A, Mastorakos G, Lambrinoudaki I, Christodoulakos G and Creatsas G: The role of the oxidative-stress in the endometriosis-related infertility. Gynecol Endocrinol 25: 75-81, 2009.

45. Jackson LW, Schisterman EF, Dey-Rao R, Browne R and Armstrong D: Oxidative stress and endometriosis. Hum Reprod 20: 2014-2020, 2005.

46. Leone Roberti Maggiore U, Scala C, Venturini PL, Remorgida V and Ferrero S: Endometriotic ovarian cysts do not negatively affect the rate of spontaneous ovulation. Hum Reprod 30 : 299-307, 2015.

47. Liu F, He L, Liu Y, Shi Y and Du H: The expression and role of oxidative stress markers in the serum and follicular fluid of patients with endometriosis. Clin Exp Obstet Gynecol 40 372-376, 2013

48. Harb HM, Gallos ID, Chu J, Harb M and Coomarasamy A The effect of endometriosis on in vitro fertilisation outcome: A systematic review and meta-analysis. BJOG 120: 1308-1320, 2013.

49. Miller JE, Ahn SH, Monsanto SP, Khalaj K, Koti M and Tayade C: Implications of immune dysfunction on endometriosis associated infertility. Oncotarget 8: 7138-7147, 2017.

50. Practice Committee of the American Society for Reproductive Medicine: Endometriosis and infertility: A committee opinion. Fertil Steril 98: 591-598, 2012

51. Pal L, Shifren JL, Isaacson KB, Chang Y, Leykin L and Toth TL: Impact of varying stages of endometriosis on the outcome of in vitro fertilization-embryo transfer. J Assist Reprod Genet 15 27-31, 1998.

52. Donnez J, Binda MM, Donnez O and Dolmans MM: Oxidative stress in the pelvic cavity and its role in the pathogenesis of endometriosis. Fertil Steril 106: 1011-1017, 2016.

53. Wang XM, Ma ZY and Song N: Inflammatory cytokines IL-6, IL-10, IL-13, TNF- $\alpha$ and peritoneal fluid flora were associated with infertility in patients with endometriosis. Eur Rev Med Pharmacol Sci 22: 2513-2518, 2018

54. Yamauchi N, Harada T, Taniguchi F, Yoshida S, Iwabe T and Terakawa N: Tumor necrosis factor-alpha induced the release of interleukin- 6 from endometriotic stromal cells by the nuclear factor-kappaB and mitogen-activated protein kinase pathways. Fertil Steril 82 (Suppl 3): S1023-S1028, 2004.

55. Falconer H, Sundqvist J, Gemzell-Danielsson K, von Schoultz B, D'Hooghe TM and Fried G: IVF outcome in women with endometriosis in relation to tumour necrosis factor and anti-Müllerian hormone. Reprod Biomed Online 18: 582-588, 2009.

56. Malvezzi H, Hernandes C, Piccinato CA and Podgaec S: Interleukin in endometriosis-associated infertility-pelvic pain: Systematic review and meta-analysis. Reproduction 158: 1-12, 2019.

57. Beste MT, Pfäffle-Doyle N, Prentice EA, Morris SN, Lauffenburger DA, Isaacson KB and Griffith LG: Molecular network analysis of endometriosis reveals a role for c-Jun-regulated macrophage activation. Sci Transl Med 6: 222ra16, 2014.

58. Agarwal A, Gupta S and Sharma RK: Role of oxidative stress in female reproduction. Reprod Biol Endocrinol 3: 28, 2005.

59. Agarwal A, Gupta S, Sekhon L and Shah R: Redox considerations in female reproductive function and assisted reproduction: From molecular mechanisms to health implications. Antioxid Redox Signal 10: 1375-1403, 2008.

60. Yoshimoto C, Iwabuchi T, Shigetomi H and Kobayashi H: Cyst fluid iron-related compounds as useful markers to distinguish malignant transformation from benign endometriotic cysts. Cancer Biomark 15: 493-499, 2015.

61. Yamaguchi K, Mandai M, Toyokuni S, Hamanishi J, Higuchi T, Takakura K and Fujii S: Contents of endometriotic cysts, especially the high concentration of free iron, are a possible cause of carcinogenesis in the cysts through the iron-induced persistent oxidative stress. Clin Cancer Res 14: 32-40, 2008

62. Kobayashi H, Yamada Y, Kanayama S, Furukawa N, Noguchi T, Haruta S, Yoshida S, Sakata M, Sado T and Oi H: The role of iron in the pathogenesis of endometriosis. Gynecol Endocrinol 25: $39-52,2009$

63. Gammella E, Recalcati S, Rybinska I, Buratti P and Cairo G: Iron-induced damage in cardiomyopathy: Oxidative-dependent and independent mechanisms. Oxid Med Cell Longev 2015: 230182, 2015.
64. Alizadeh M, Mahjoub S, Esmaelzadeh S, Hajian K, Basirat Z and Ghasemi M: Evaluation of oxidative stress in endometriosis: A case-control study. Caspian J Intern Med 6: 25-29, 2015.

65. Montoya-Estrada A, Coria-García CF, Cruz-Orozco OP, Aguayo-González P, Torres-Ramos YD, Flores-Herrera H, Hicks JJ, Medina-Navarro R and Guzmán-Grenfell AM: Increased systemic and peritoneal oxidative stress biomarkers in endometriosis are not related to retrograde menstruation. Redox Rep 24: 51-55, 2019.

66. Benaglia L, Paffoni A, Mangiarini A, Restelli L, Bettinardi N, Somigliana E, Vercellini P and Fedele L: Intrafollicular iron and ferritin in women with ovarian endometriomas. Acta Obstet Gynecol Scand 94: 646-653, 2015

67. Karuppagounder SS, Alim I, Khim SJ, Bourassa MW, Sleiman SF, John R, Thinnes CC, Yeh TL, Demetriades M, Neitemeier S, et al: Therapeutic targeting of oxygen-sensing prolyl hydroxylases abrogates ATF4-dependent neuronal death and improves outcomes after brain hemorrhage in several rodent models. Sci Transl Med 8: 328ra29, 2016.

68. Yang CH, Kung TA and Chen PJ: Differential alteration in reproductive toxicity of medaka fish on exposure to nanoscale zerovalent iron and its oxidation products. Environ Pollut 252: 1920-1932, 2019

69. Singh AK, Chattopadhyay R, Chakravarty B and Chaudhury K: Markers of oxidative stress in follicular fluid of women with endometriosis and tubal infertility undergoing IVF. Reprod Toxicol 42: 116-124, 2013.

70. Fujimoto Y, Imanaka S, Yamada Y, Ogawa K, Ito F, Kawahara N, Yoshimoto $\mathrm{C}$ and Kobayashi $\mathrm{H}$ : Comparison of redox parameters in ovarian endometrioma and its malignant transformation. Oncol Lett 16: 5257-5264, 2018.

71. Niiro E, Kawahara N, Yamada Y, Yoshimoto C, Shimada K, Sudo T and Kobayashi H: Immunohistochemical expression of $\mathrm{CD} 44 \mathrm{v} 9$ and $8-\mathrm{OHdG}$ in ovarian endometrioma and the benign endometriotic lesions adjacent to clear cell carcinoma. J Obstet Gynaecol Res 45: 2260-2266, 2019.

72. Da Broi MG, Jordão AA Jr, Ferriani RA and Navarro PA: Oocyte oxidative DNA damage may be involved in minimal/mild endometriosis-related infertility. Mol Reprod Dev 85: 128-136, 2018.

73. Da Broi MG, de Albuquerque FO, de Andrade AZ, Cardoso RL, Jordão Junior AA and Navarro PA: Increased concentration of 8-hydroxy-2'-deoxyguanosine in follicular fluid of infertile women with endometriosis. Cell Tissue Res 366: 231-342, 2016.

74. Santanam N, Zoneraich N and Parthasarathy S: Myeloperoxidase as a potential target in women with endometriosis undergoing IVF. Reprod Sci 24: 619-626, 2017.

75. Seino T, Saito H, Kaneko T, Takahashi T, Kawachiya S, Kurachi H: Eight-hydroxy-2'-deoxyguanosine in granulosa cells is correlated with the quality of oocytes and embryos in an in vitro fertilization-embryo transfer program. Fertil Steril 77: 1184-1190, 2002

76. Agarwal A, Gupta S and Sharma R: Oxidative stress and its implications in female infertility-a clinician's perspective. Reprod Biomed Online 11: 641-650, 2005

77. Choi YS, Cho S, Seo SK, Park JH, Kim SH and Lee BS: Alteration in the intrafollicular thiol-redox system in infertile women with endometriosis. Reproduction 149: 155-162, 2015.

78. Conrad M, Kagan VE, Bayir H, Pagnussat GC, Head B, Traber MG and Stockwell BR: Regulation of lipid peroxidation and ferroptosis in diverse species. Genes Dev 32: 602-619, 2018

79. Yamada Y, Uchiyama T, Ito F, Kawahara N, Ogawa K, Obayashi C and Kobayashi H: Clinical significance of M2 macrophages expressing heme oxygenase-1 in malignant transformation of ovarian endometrioma. Pathol Res Pract 215: 639-643, 2019.

80. Akasaka J, Uekuri C, Shigetomi H, Koike M and Kobayashi H: Hepatocyte nuclear factor (HNF)-1 $\beta$ and its physiological importance in endometriosis. Biomed Rep 1: 13-17, 2013.

81. Stockwell BR, Friedmann Angeli JP, Bayir H, Bush AI, Conrad M, Dixon SJ, Fulda S, Gascón S, Hatzios SK, Kagan VE, et al: A regulated cell death nexus linking metabolism, redox biology, and disease. Cell 171: 273-285, 2017.

82. Ng SW, Norwitz SG, Taylor HS and Norwitz ER: Endometriosis: The role of iron overload and ferroptosis. Reprod Sci, Feb 19, 2020 (Epub ahead of print).

83. Notarstefano V, Gioacchini G, Byrne HJ, Zacà C, Sereni E, Vaccari L, Borini A, Carnevali O and Giorgini E: Vibrational characterization of granulosa cells from patients affected by unilateral ovarian endometriosis: New insights from infrared and Raman microspectroscopy. Spectrochim Acta A Mol Biomol Spectrosc 212: 206-214, 2019. 
84. Nishihara T, Matsumoto $\mathrm{K}$, Hosoi $\mathrm{Y}$ and Morimoto $\mathrm{Y}$ : Evaluation of antioxidant status and oxidative stress markers in follicular fluid for human in vitro fertilization outcome. Reprod Med Bio 17: 481-486, 2018.

85. Kumar S, Mishra V, Thaker R, Gor M, Perumal S, Joshi P, Sheth H, Shaikh I, Gautam AK and Verma Y: Role of environmental factors \& oxidative stress with respect to in vitro fertilization outcome Indian J Med Res 148 (Suppl): S125-S133, 2018.

86. Velthut A, Zilmer M, Zilmer K, Kaart T, Karro H and Salumets A: Elevated blood plasma antioxidant status is favourable for achieving IVF/ICSI pregnancy. Reprod Biomed Online 26: 345-352, 2013.

87. Donabela FC, Meola J, Padovan CC, de Paz CC and Navarro PA: Higher SOD1 gene expression in cumulus cells from infertile women with moderate and severe endometriosis. Reprod Sci 22: $1452-1460,2015$

88. Nakagawa $K$, Hisano M, Sugiyama $R$ and Yamaguchi $K$ : Measurement of oxidative stress in the follicular fluid of infertility patients with an endometrioma. Arch Gynecol Obstet 293: 197-202, 2016

89. Jozwik M, Wolczynski S, Jozwik M and Szamatowicz M Oxidative stress markers in preovulatory follicular fluid in humans. Mol Hum Reprod 5: 409-413, 1999.

90.Zilka O, Shah R, Li B, Friedmann Angeli JP, Griesser M, Conrad M and Pratt DA: On the mechanism of cytoprotection by ferrostatin-1 and liproxstatin-1 and the role of lipid peroxidation in ferroptotic cell death. ACS Cent Sci 3: 232-243, 2017.

91. Matsuzaki S and Darcha C: Antifibrotic properties of epigallocatechin-3-gallate in endometriosis. Hum Reprod 29: 1677-1687, 2014

92. Defrère S, Van Langendonckt A, Vaesen S, Jouret M, González Ramos R, Gonzalez D and Donnez J: Iron overload enhances epithelial cell proliferation in endometriotic lesions induced in a murine model. Hum Reprod 21: 2810-2816, 2006.

93. Showell MG, Mackenzie-Proctor R, Jordan V and Hart RJ Antioxidants for female subfertility. Cochrane Database Syst Rev 7: CD007807, 2017.

94. Ito F, Yamada Y, Shigemitsu A, Akinishi M, Kaniwa H, Miyake R, Yamanaka S and Kobayashi H: Role of oxidative stress in epigenetic modification in endometriosis. Reprod Sci 24: 1493-1502, 2017

95. Niu Y, DesMarais TL, Tong Z, Yao Y and Costa M: Oxidative stress alters global histone modification and DNA methylation. Free Radic Biol Med 82: 22-28, 2015.

96. Sun Q, Ding D, Liu X and Guo SW: Tranylcypromine, a lysine-specific demethylase 1 (LSD1) inhibitor, suppresses lesion growth and improves generalized hyperalgesia in mouse with induced endometriosis. Reprod Biol Endocrinol 14: 17, 2016

97. Guo SW, Ding D, Shen M and Liu X: Dating endometriotic ovarian cysts based on the content of cyst fluid and its potential clinical implications. Reprod Sci 22: 873-883, 2015.

98. Capobianco A, Cottone L, Monno A, Manfredi AA Rovere-Querini P: The peritoneum: Healing, immunity, and diseases. J Pathol 243: 137-147, 2017.

99. Sanchez AM, Viganò P, Somigliana E, Panina-Bordignon $\mathrm{P}$ Vercellini $\mathrm{P}$ and Candiani $\mathrm{M}$ : The distinguishing cellular and molecular features of the endometriotic ovarian cyst: From pathophysiology to the potential endometrioma-mediated damage to the ovary. Hum Reprod Update 20: 217-230, 2014.

100.Zeng X, Yue Z, Gao Y, Jiang G, Zeng F, Shao Y, Huang J, Yin M and Li Y: NR4A1 is Involved in fibrogenesis in ovarian endometriosis. Cell Physiol Biochem 46: 1078-1090, 2018.

101. Viganò P, Ottolina J, Bartiromo L, Bonavina G, Schimberni M, Villanacci R and Candiani M: Cellular components contributing to fibrosis in endometriosis: A literature review. J Minim Invasive Gynecol 27: 287-295, 2020.

102. Seki E and Schwabe RF: Hepatic inflammation and fibrosis: Functional links and key pathways. Hepatology 61: 1066-1079, 2015.

103. Zhang Q, Duan J, Liu X and Guo SW: Platelets drive smooth muscle metaplasia and fibrogenesis in endometriosis through epithelial-mesenchymal transition and fibroblast-to-myofibroblast transdifferentiation. Mol Cell Endocrinol 428: 1-16, 2016.

104. Wynn TA and Ramalingam TR: Mechanisms of fibrosis: Therapeutic translation for fibrotic disease. Nat Med 18 1028-1040, 2012

105. Guo SW, Du Y and Liu X: Platelet-derived TGF- $\beta 1$ mediates the down-modulation of NKG2D expression and may be responsible for impaired natural killer (NK) cytotoxicity in women with endometriosis. Hum Reprod 31: 1462-1474, 2016.

106. Wynn TA and Vannella KM: Macrophages in tissue repair, regeneration, and fibrosis. Immunity 44: 450-462, 2016.
107. Zhou Y, Que KT, Zhang Z, Yi ZJ, Zhao PX, You Y, Gong JP and Liu ZJ: Iron overloaded polarizes macrophage to proinflammation phenotype through ROS/acetyl-p53 pathway. Cancer Med 7: 4012-4022, 2018

108. Agoro R, Taleb M, Quesniaux VFJ and Mura C: Cell iron status influences macrophage polarization. PLoS One 13: e0196921, 2018.

109. Ngô C, Chéreau C, Nicco C, Weill B, Chapron C and Batteux F: Reactive oxygen species controls endometriosis progression. Am J Pathol 175: 225-234, 2009.

110. Barcena de Arellano ML, Arnold J, Lang H, Vercellino GF, Chiantera V, Schneider A and Mechsner S: Evidence of neurotrophic events due to peritoneal endometriotic lesions. Cytokine 62: 253-261, 2013.

111. Wilson RB: Hypoxia, cytokines and stromal recruitment: Parallels between pathophysiology of encapsulating peritoneal sclerosis, endometriosis and peritoneal metastasis. Pleura Peritoneum 3: 20180103, 2018

112. Daftary GS, Zheng Y, Tabbaa ZM, Schoolmeester JK, Gada RP, Grzenda AL, Mathison AJ, Keeney GL, Lomberk GA and Urrutia R: A novel role of the Sp/KLF transcription factor KLF11 in arresting progression of endometriosis. PLoS One 8: e60165, 2013.

113. Delaney AA, Khan Z, Zheng Y, Correa LF, Zanfagnin V, Shenoy CC, Schoolmeester JK, Saadalla AM, El-Nashar S, Famuyide AO, et al: KLF10 mediated epigenetic dysregulation of epithelial CD40/CD154 promotes endometriosis. Biol Reprod 95: 62, 2016.

114. Maia H Jr, Haddad C, Coelho G and Casoy J: Role of inflammation and aromatase expression in the eutopic endometrium and its relationship with the development of endometriosis. Womens Health (Lond) 8: 647-658, 2012.

115. Keskin HL, Sirin YS, Keles H, Turgut O, Ide T and Avsar AF: The aromatase inhibitor letrozole reduces adhesion formation after intraperitoneal surgery in a rat uterine horn model. Eur J Obstet Gynecol Reprod Biol 167: 199-204, 2013.

116. Xiao X, Senavirathna LK, Gou X, Huang C, Liang Y and Liu L: EZH2 enhances the differentiation of fibroblasts into myofibroblasts in idiopathic pulmonary fibrosis. Physiol Rep 4: pii: e12915, 2016.

117. Zhang Q, Dong P, Liu X, Sakuragi N and Guo SW: Enhancer of Zeste homolog 2 (EZH2) induces epithelial-mesenchymal transition in endometriosis. Sci Rep 7: 6804, 2017.

118. Ahmed SM, Luo L, Namani A, Wang XJ and Tang X: Nrf2 signaling pathway: Pivotal roles in inflammation. Biochim Biophys Acta Mol Basis Dis 1863: 585-597, 2017.

119. Marcellin L, Santulli P, Chouzenoux S, Cerles O, Nicco C, Dousset B, Pallardy M, Kerdine-Römer S, Just PA, Chapron C and Batteux F: Alteration of Nrf2 and glutamate cysteine ligase expression contribute to lesions growth and fibrogenesis in ectopic endometriosis. Free Radic Biol Med 110: 1-10, 2017

120. Kefaloyianni E, Muthu ML, Kaeppler J, Sun X, Sabbisetti V, Chalaris A, Rose-John S, Wong E, Sagi I, Waikar SS, et al: ADAM17 substrate release in proximal tubule drives kidney fibrosis. JCI Insight 1: pii: E87023, 2016

121. $\mathrm{Hu} \mathrm{B}$ and Phan SH: Notch in fibrosis and as a target of anti-fibrotic therapy. Pharmacol Res 108: 57-64, 2016.

122. Ruiz LA, Báez-Vega PM, Ruiz A, Peterse DP, Monteiro JB, Bracero N, Beauchamp P, Fazleabas AT and Flores I: Dysregulation of lysyl oxidase expression in lesions and endometrium of women with endometriosis. Reprod Sci 22: 1496-1508, 2015.

123. Kubes P and Mehal WZ: Sterile inflammation in the liver. Gastroenterology 143: 1158-1172, 2012.

124. Cao Y, Liu X and Guo SW: Plasma high mobility group Box 1 (HMGB1), osteopontin (OPN), and hyaluronic acid (HA) as admissible biomarkers for endometriosis. Sci Rep 9: 9272 , 2019.

125. Sharma I, Dhawan V, Saha SC, Rashmi B and Dhaliwal LK Implication of the RAGE-EN-RAGE ax is in endometriosis. In J Gynaecol Obstet 110: 199-202, 2010.

126. Zondervan KT, Becker CM, Koga K, Missmer SA, Taylor RN and Viganò P: Endometriosis. Nat Rev Dis Primers 4: 9, 2018.

his work is licensed under a Creative Commons Attribution-NonCommercial-NoDerivatives 4.0 International (CC BY-NC-ND 4.0) License. 